\title{
Knowledge retrieval for autonomous agents
}

\author{
E. Szczerbicki \\ The University of Newcastle \\ Newcastle, NSW 2308, Australia. \\ Tel: (49) 216209 . Fax: (49) 216946. \\ e-mail: meeslcc.newcastle.edu.au
}

\begin{abstract}
The paper addresses the problem of developing the domain knowledge base for autonomous manufacturing agents. In an organisational context, autonomous agents consist of elements (people, machines, robots, etc.) tied by the flow of information between an agent and its external environment as well as within an agent. Various tools can be used to develop and evaluate such an information flow. In the paper, the application of mathematical modelling, decision trees, and neural networks is investigated and illustrated with examples.
\end{abstract}

\section{Keywords}

Information flow, production rules, knowledge acquisition, autonomous manufacturing agents, decision trees, neural networks

\section{AUTONOMOUS AGENTS MATHEMATICAL MODELLING AND REPRESENTATION}

Structuring an information flow for a manufacturing agent belongs to the functional area of management and forms the basis for further agent integration. Mathematical models are designed to describe, understand, and finally support processes and activities that are primarily intellectual. In other words, models are developed mainly to create knowledge. The formal model is presented that can be used in creation of knowledge connected with an information flow evaluation in autonomous systems.

A general discussion of some principles of the functioning of autonomous systems and the role of information flow in an "information society" is included in Zeigler and Rozenblit (1990) and in Richards and Gupta (1985). The problem of information modelling for the 
integration of computerised manufacturing agents is discussed in Hsu and Rattner (1990). Mathematical representation of autonomous systems functioning in various decision situations described by the characteristics of external and internal environments is presented in Szczerbicki (1994). In this paper the approach developed earlier is used as the basis for quantitative and non-quantitative agent representation and the retrieval of knowledge concerning agent functioning.

\subsection{Information flow for autonomous agents: principles and tools for modeling and evaluation}

There are three components which are the key to understanding the functioning of an autonomous system. They are information structure, external environment, and internal environment. The growth of interest in these three components defining autonomous systems in all kinds of manufacturing organisations that strive for integration through the flow of information is apparent.

Formal representation of decision-making processes represents the core of the generalised model of autonomous agents functioning. Since most autonomous systems involve decision analysis with incomplete knowledge under uncertainty, the best alternative is very often defined, by assuming that the description of the environment is known statistically, as the one that maximises the expected utility. Let $\mathbf{A}$ represent the set of possible actions which can be undertaken by the element of an agent, $\mathbf{Z}$ the set of corresponding consequences, and $\mathbf{X}$ random variables describing the actual state of the external environment. It can be assumed that $z=\mathrm{f}(a, x)$ as the particular consequence $(z)$ depends usually on an action $(a)$ undertaken in the particular state of the environment $(x)$. On the other hand, the decision about particular action depends on information that is available about the state of the environment. If $B$ stands for the decision function, we have $a=\beta(d)$ where $d$ represents information. It can be shown that, after considering certain correlation between information, action, and energy, we have:

$\mathbf{f}(a, x)=\mathbf{B}_{0}-2 \mathbf{B}^{\mathrm{T}} \mathbf{A}+\mathbf{A}^{\mathrm{T}} \mathbf{Q A}$,

where $\mathbf{B}_{0}=\mathrm{b}_{0}(x), \mathbf{A}=\left[a_{i}\right], \mathbf{B}=\left[b_{i}(x)\right]$, symmetric matrix $\mathbf{Q}=\left[q_{i j}\right](i, j=1,2, \ldots, n)$ and $n$ represents the number of elements of an agent. Minimum of (1) exists if $\mathbf{A}^{\mathrm{T}} \mathbf{Q A}$ is positively defined.

For an $n$-element agent we have:

$$
\beta_{\mathrm{i}}\left(d_{i}\right)+\sum_{j \neq i} q_{i j} \mathrm{E}\left[\beta_{\mathrm{j}}\left(d_{j}\right) \mid d_{i}\right]=\mathrm{E}\left(b_{i} \mid d_{i}\right),
$$

where $i, j=1,2, \ldots, n$.

Formalisation of agent decision making process expressed by (2) is a tool necessary for modeling and evaluation of information flow in an autonomous system. Information flow connects agent elements with the external environment described by random variables $\mathbf{X}$. For our purposes we define information as knowledge about realisation $\mathbf{X}$ and the above connection is represented by information structure. This structure is modelled by matrix $\mathbf{C}$ in which $c_{i j}=1$ if the $i$ th element has obtained (either by observation - sensoring, monitoring - or 
communication) information about the $j$ th variable $\mathbf{X}$ realisation (if $c_{i j}=0$ it has not got it). The $i$ th variable $\mathbf{X}$ realisation can be observed (sensored, monitored) only by the $i$ th element of the agent. It can be informed about other realisations only when communication (information exchange) inside the agent is organised.

The value of information structure defined above is given as:

$V C=\min \mathbf{E}[\mathbf{f}(\mathbf{A}, \mathbf{X}) \mid \mathbf{C} 0]-\min E[f(\mathbf{A}, \mathbf{X}) \mid \mathbf{C}]$,

where $\min \mathrm{E}[\mathrm{f}(\mathbf{A}, \mathbf{X}) \mid \mathrm{C} 0]$ represents the utility of information structure $\mathbf{C 0}$ in which $c_{i j}=0$ for each $i$ and $j$. Using (2) the $V C$ can be represented by:

$V C=\mathrm{E}\left[\mathbf{b}^{\mathrm{T}} \beta\right]$.

\subsection{Model-based generation of knowledge}

With the mathematical tools presented shortly above it is possible to carry out various simulations of agent functioning in different decision situations. Such simulations help us to understand the role of various parameters describing agent external and internal environments in an information flow evaluation. The results of the model-based simulations can be formulated as IF...AND...THEN rules that provide useful knowledge about autonomous agents functioning in static and dynamic environments. Generation of such knowledge is an important factor in successful implementation of integration based on the flow of information. In static environment such factors as correlation, interaction, number of agent elements, and incompleteness of information were considered. In dynamic environment the corresponding rules consider the character of dynamics (stable, Brownian, and explosive), and delay. Examples of such rules are presented next.

Samples of production rules for static environment.

\section{RULE 12}

IF an external environment of an autonomous agent is static,

AND there is an interaction in the internal environment,

AND the relationship between variables describing the external environment is of statistical character,

THEN information structure should include observation (sensoring) and communication.

\section{RULE 13}

IF an external environment of an autonomous agent is static,

AND the relationship between variables describing the external environment is given by function dependence,

THEN communication between agent elements does not affect the value of information structure; information flow should be restricted to observation (sensoring).

Samples of production rules for dynamic environment.

\section{RULE 14}

IF an external environment of an autonomous group is described by stochastic process,

AND information is not delayed,

AND the external environment is described by Brownian movement,

THEN the value of information structures increases proportionally with the increase of time. 


\section{RULE 17}

IF an external environment of an autonomous group is described by stochastic process,

AND information is delayed,

AND stochastic process is dependent,

AND the external environment is stable,

THEN the losses caused by delayed information stabilise with increasing value of delay.

\section{NONQUANTITATIVE TOOLS AND TECHNIQUES}

\subsection{Decision tree classifiers}

Decision tree classifiers are used successfully in many diverse areas. Their most important feature is the capability of capturing descriptive decisionmaking knowledge from the supplied data (Safavian and Landgrebe 1991). Decision tree can be generated from training sets. The procedure for such generation based on the set of objects (S), each belonging to one of the classes $\mathbf{C}_{1}, \mathbf{C}_{2}, \ldots, \mathbf{C}_{\mathbf{k}}$ is as follows (Quinlan 1990):

Step 1. If all the objects in $\mathbf{S}$ belong to the same class, for example $\mathbf{C}_{i}$, the decision tree for $\mathbf{S}$ consists of a leaf labelled with this class.

Step 2. Otherwise, let T be some test with possible outcomes $O_{1}, O_{2}, \ldots, O_{n}$. Each object in $\mathbf{S}$ has one outcome for $T$ so the test partitions $\mathbf{S}$ into subsets $\mathbf{S}_{1}, \mathbf{S}_{2}$, $\ldots \mathbf{S}_{\mathbf{n}}$ where each object in $\mathbf{S}_{\mathrm{i}}$ has outcome $O_{i}$ for T. T becomes the root of the decision tree and for each outcome $O_{i}$ we build a subsidiary decision tree by invoking the same procedure recursively on the set $\mathbf{S}_{\mathbf{i}}$.

The above procedure is applied to the training set of objects in Table 1. The training sets are delivered from the analysis based on the quantitative model of agent functioning (Szczerbicki 1994). Each object is described by the relating attributes and belongs to one of the agent decision classes exchange information ("yes" in the last column) or do_not_exchange_information ("no" in the last column).

Table 1 Training set for agent functioning

\begin{tabular}{|c|c|c|c|c|c|}
\hline $\begin{array}{l}\text { external } \\
\text { environment }\end{array}$ & $\begin{array}{l}\text { internal } \\
\text { environment }\end{array}$ & $\begin{array}{l}\text { type of } \\
\text { dynamics }\end{array}$ & correlation & $\begin{array}{l}\text { delay of } \\
\text { information }\end{array}$ & decision \\
\hline static & independent_actions & 0 & 0 & 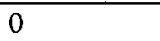 & no \\
\hline static & independent_actions & 0 & 0.7 & 1 & no \\
\hline dynamic & dependent_actions & 1.5 & -0.5 & 1 & yes \\
\hline static & dependent_actions & 0 & 0 & 0 & yes \\
\hline static & independent_actions & 0 & 1 & 2 & no \\
\hline static & dependent_actions & 0 & 0.5 & 2 & yes \\
\hline static & dependent actions & 0 & -1 & 3 & no \\
\hline static & independent_actions & 0 & -1 & 0 & no \\
\hline static & dependent_actions & 0 & 0.9 & 1 & yes \\
\hline static & dependent actions & 0 & 1 & 1 & no \\
\hline
\end{tabular}

Suppose, for the sake of simplicity, that we are interested in decision making situations involving static environment only. When for this case the set is partitioned by testing on 
internal_environment and then on correlation, the resulting structure is equivalent to the decision tree shown in Figure 1.

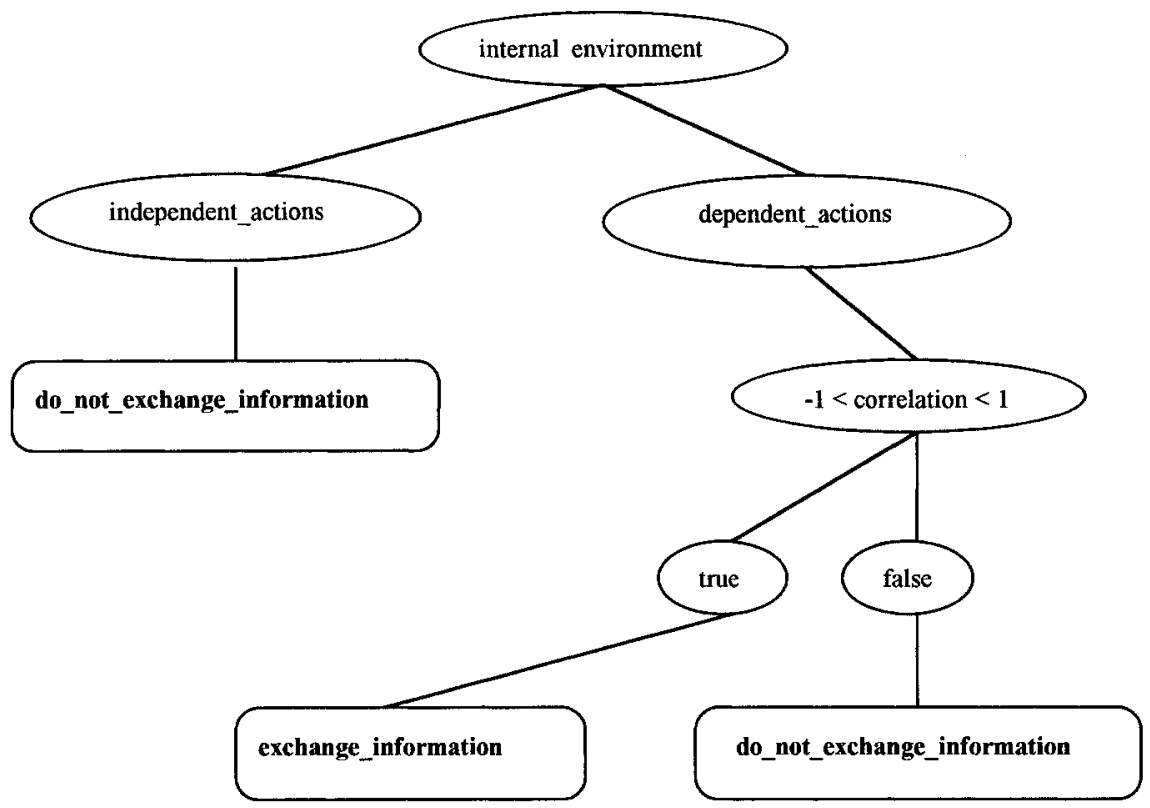

Figure 1 Decision tree classifier for agent decisionmaking.

The following rules can be delivered from Figure 1

\section{RULE 1}

IF an external environment of an agent is static

AND it is described by random variables

AND there is no interaction in the internal environment

THEN communication (exchange of information) between agent elements is

RULE 2 not necessary

IF an external environment of an agent is static

AND it is described by random variables

AND there is interaction in the internal environment

AND the relationship between variables describing the external environment is of statistical character

THEN exchange of information between agent elements should be organised

\section{RULE 3}

IF an external environment of an agent is static

AND it is described by random variables 
AND there is interaction in the internal environment

AND the relationship between variables describing the external environment is given by function dependence

THEN exchange of information between agent elements is not necessary

The simple tree in Figure 1 has been used to retrieve some of the knowledge concerning the functioning of an agent in static environment. The decision tree, once developed, can support decision situations that are not covered by the training set. That is why the production rules can be formulated as a generalised statements. As it has been illustrated the use of decision trees is simple and as effective as the analysis based on a rigorous mathematical model (the production rules formulated above are exactly the same as the Rules 4,5, and 6 given in Szczerbicki 1994). Another tool that can be of much help in the process of knowledge retrieval is the technique based on connectionist systems.

\subsection{Connectionist systems}

Neural networks which learn mappings between sets of patterns are called mapping neural networks (Maren et.al.). A key property of mapping networks is their ability to produce reasonable output vectors for input patterns outside of the set of training examples (please note the similarity to the decision tree classifiers). The above is especially important in areas such as discussed in this paper, i.e. areas for which it is possible to develop only a very limited number of IF...THEN rules and thus also to make inferences only for a very limited number of decision situations.

Problem solving tasks, such as information structure development for an autonomous agent, may be considered pattern classification tasks. The system analyst learns mappings between input patterns, consisting of characteristics of agent's external and internal environment, and output patterns, consisting of information structures to apply to these characteristics. Thus, neural networks (neural-based expert systems) offer a promising solution for automating the learning process of the analyst.

As we already know, systems analyst, while developing an information structure for an agent, transforms certain characteristics of an agent into recommendations concerning the flow of information. These characteristics represent the input for the system and their full description (for both static and dynamic environments) includes 5 parameters: correlation in the external environment $(r)$, dynamics $(t)$, interaction in the internal environment $(q)$, delay $(d)$, and type of the process describing the external environment (w). Output consists of the following decisions (recommendations): (i) observation (or sensoring) should be present, and (ii) exchange of information should be present. Please note that the decision concerning sensoring of information is added for this case (it was not considered in Section 2.1). An input portion together with an output portion of the data represents a training pair. The training pairs were used to train a 5-10-2 neural network.

The target values for each output node were normalised in such a way that the maximum target for each node received a value of 0.75 and the minimum target for each node received a value of 0.25 . The training values for each input node were identically normalised. The learning rate and momentum term of 0.9 were used in the network. The network was trained using error back propagation procedure with a training tolerance of $5 \%$. The network was 
considered trained if, for all training pairs and output nodes, |(desired output - actual output)/(desired output) $\mid<$ tolerance.

After training, additional characteristics of an agent were generated for use of the network. Five sets of characteristics were submitted to the network. In response, the network suggested five information flow recommendations. As an example, Table 2 presents two sets of characteristics submitted and the obtained recommendations after the trained network has been used. For the first input set (no. 1 in Table 2) the network recommends decentralised information structure (only observation, no exchange of information). For the second, full information structure is recommended (observation and exchange). In both cases the recommendations agree with the IF ... AND ... THEN rules discussed in Szczerbicki (1994).

Table 2 The use of the trained network

\begin{tabular}{|c|c|c|c|c|}
\hline no & value & description & observation & exchange \\
\hline 1 & $\begin{array}{l}t=0 \\
q=0.01 \\
d=0 \\
w=0\end{array}$ & $\begin{array}{l}\text { strong relationship } \\
\text { between variables } \\
\text { describing external } \\
\text { environment } \\
\text { external environment } \\
\text { is static } \\
\text { there is no } \\
\text { interaction in } \\
\text { internal environment } \\
\text { information is not } \\
\text { delayed } \\
\text { process is } \\
\text { independent }\end{array}$ & yes & no \\
\hline 2 & $\begin{array}{l}t=0 \\
q=0.90 \\
d=0 \\
w=0\end{array}$ & $\begin{array}{l}\text { weak relationship } \\
\text { between variables } \\
\text { describing external } \\
\text { environment } \\
\text { external environment } \\
\text { is static } \\
\text { there is interaction } \\
\text { in internal environment } \\
\text { information is } \\
\text { not delayed } \\
\text { process is independent }\end{array}$ & yes & yes \\
\hline
\end{tabular}




\section{CONCLUSIONS}

For the decision making process supporting agents functioning and information flow development, the knowledge domain includes many abstract concepts. The formal quantitative model can be used to generate some examples of the above domain knowledge. Quantitative models of an information flow evaluation, however, are often too complex to serve as the tools useful in the knowledge retrieval process. In the paper the preliminary results of two non-quantitative procedures applied in the process of knowledge acquisition have been presented and illustrated with examples. The procedures, decision trees and neural networks, show the potential for use in reasoning and retrieval of knowledge describing the flow of information between an agent and its external environment as well as within an agent. It was shown that the techniques applied are able to provide general knowledge about agent functioning in static and dynamic external environments. Both techniques, back propagation algorithm and decision tree classifier, illustrate the ease and appropriateness of such methods for dealing with implicit knowledge and also provide a model for extension into other expert domains.

\section{REFERENCES}

Hsu, C. and Rattner, L. (1990) Information modeling for computerised manufacturing. IEEE Transactions on Systems, Man, and Cybernetics, 20, 758-776.

Maren, A.J., Harston, C.T. and Pap, R.M. (1990) Handbook of neural computing applications. Academic Press, New York.

Quinlan, J.R. (1990) Decision trees and decision making. IEEE Transactions on Systems, Man, and Cybernetics, 20, 339-346.

Richards, L.D. and Gupta, S.K. (1985) The systems approach in an information society: a reconsideration. Journal of Operational Research Society, 36, 833-843.

Safavian, S. and Landgrebe, D. (1991) A survey of decision tree classifier methodology. IEEE Transactions on Systems, Man, and Cybernetics, 21, 660-674.

Szczerbicki, E. (1994) Model-based generation of knowledge for autonomous systems. International Journal of Systems Science, 25, 453-472.

Zeigler, B. and Rozenblit, J. (ed.) (1990) AI, Simulation and Planning in High Autonomy Systems. IEEE Computer Society Press, Los Alamitos. 